\title{
PRESSURE EFFECTS ON THE TEMPERATURE SENSITIVITY OF FIBER BRAGG GRATINGS
}

\author{
Meng-Chou $\mathrm{Wu}$ \\ NASA Langley Research Center, MS 231, Hampton, Virginia, USA, 23681-219
}

\begin{abstract}
A 3-dimensional physical model was developed to relate the wavelength shifts resulting from temperature changes of fiber Bragg gratings (FBGs) to the thermal expansion coefficients, Young's moduli of optical fibers, and thicknesses of coating polymers. Using this model the Bragg wavelength shifts were calculated and compared with the measured wavelength shifts of FBGs with various coating thickness for a finite temperature range. There was a discrepancy between the calculated and measured wavelength shifts. This was attributed to the refractive index change of the fiber core by the thermally induced radial pressure. To further investigate the pressure effects, a small diametric load was applied to a FBG and Bragg wavelength shifts were measured over a temperature range of 4.2 to $300 \mathrm{~K}$.
\end{abstract}

Keywords: Fiber Bragg Gratings, Temperature Sensing, Cryogenic Temperatures, Pressure Effects PACS: 42.81.Pa,

\section{INTRODUCTION}

Fiber Bragg gratings (FBGs) have been extensively proposed and utilized for health monitoring systems of aerospace structures and materials as well as many other applications [1,2]. Compared to other sensors, FBGs have the advantages of being lightweight and flexible, and requiring simpler wiring especially for distributed sensing. Most efforts have focused on using distributed fiber optic strain sensing systems, with limited consideration given to temperature sensing systems. In general, strain response of FBGs is linear for a broad range of strain and independent of temperature [3]. The temperature response of FBGs is much more complicated. The temperature sensitivity of FBGs is moderate and relatively linear for temperatures above $273 \mathrm{~K}$. However, it becomes smaller and more nonlinear as the temperature decreases further below $273 \mathrm{~K}$ and much smaller for cryogenic temperatures.

It has been suggested that FBGs be embedded in or bonded to substrates, e.g., Teflon to enhance their temperature sensitivity [4]. These substrates have larger thermal expansion coefficients than silica fibers. While the enhancement of FBG temperature sensitivity is significant using these techniques, the flexibility of the optical fiber is sacrificed because of the need for bonding to a rigid substrate. This is particularly unsuitable for distributed sensing with multiple sensors. Another alternative is using FBGs with polymer coatings of finite thickness, which give smaller enhancement of temperature sensitivity but do not compromise the flexibility of the optical fibers. 
This study investigated the effects of polymer coatings on FBG temperature sensitivity. A 3-dimensional physical model was developed to relate the temperature sensitivity of FBGs to coating thickness. Trends predicted by the physical model were compared to experimental results. There was a discrepancy between the calculated and measured wavelength shifts. This was attributed to effects of the thermally induced radial pressure in the fibers. To further investigate the pressure effects the pressurized fiber Bragg gratings (PFBGs) were introduced. PFBGs can be obtained by applying a small diametric load to regular FBGs. In this study, a pressure-induced transition of the PFBGs occurred at approximately $200 \mathrm{~K}$ and resulted in higher temperature sensitivity for PFBGs than FBGs at cryogenic temperatures. This intriguing phenomenon was independent of the polymer coatings and attributed to a substantial change of the refractive index of the fiber core.

\section{THEORY}

\section{Thermally Induced Effects of a Polymer Coated Fiber}

In general a fiber Bragg grating can be characterized by its Bragg wavelength, which is the center wavelength of the light reflected from the grating. The Bragg wavelength is given as

$$
\lambda_{\mathrm{B}}=2 \mathrm{n}_{\mathrm{eff}} \Lambda
$$

where $\mathrm{n}_{\text {eff }}$ is the effective refractive index of the fiber core and $\Lambda$ the grating period. For a fiber Bragg grating coated with polymer, a change in the temperature causes a change in the grating period due to not only the thermal expansion of the fiber but also the strain, $\delta l / l$, induced by thermal expansion of the coating polymer. In addition, the refractive index of the fiber core changes because of the thermo-optic effect. Combining all the above effects, the shift in the Bragg wavelength due to finite temperature change, $\Delta T$, is given as

$$
\delta \lambda_{\mathrm{B}} / \lambda_{\mathrm{B}}=\alpha_{\mathrm{s}} \Delta T+\left(1-\mathrm{p}_{\mathrm{e}}\right) \delta l / l+\delta \mathrm{n}_{\mathrm{eff}} / \mathrm{n}_{\mathrm{eff}},
$$

where $\alpha_{\mathrm{s}}$ is the thermal expansion coefficient, and $\mathrm{p}_{\mathrm{e}}$ the photoelastic constant of the fiber, i.e., silica glass.

To estimate the contribution of the second term in Eq. (2) to the total wavelength shift, a complete 3-dimensional model is needed to calculate the thermally induced strain, $\delta l / l$, which should be expressed by all of the physical constants and the geometry as shown in Fig.1. Details of the derivation are in the Appendix. $\delta l / l$ can be obtained as

where

$$
\delta l / l=\eta\left(\alpha_{\mathrm{p}}-\alpha_{\mathrm{s}}\right) \Delta T
$$

$$
\eta \equiv\left[1+\frac{\mathrm{E}_{\mathrm{s}}\left(1+2 v_{p} \zeta\right)}{\gamma \mathrm{E}_{\mathrm{p}}\left(1-2 v_{s} \zeta\right)}\right]^{-1}
$$

$\gamma$ is the ratio of the cross-sectional area, $A_{p}$, of the polymer with a radial thickness $\mathrm{t}$, to the cross-sectional area, $A_{s}$, of the silica fiber with a radius of $r_{s}$, and $\xi$ is defined as the ratio of the induced radial stress, $\mathrm{p}_{\mathrm{r}}$, to the axial stress along the fiber, $\sigma_{z z}^{s}$, 


$$
\zeta \equiv \frac{\mathrm{p}_{\mathrm{r}}}{\sigma_{z z}^{s}}=\left[\left(\frac{1+v_{p}}{\gamma \mathrm{E}_{\mathrm{p}}}+\frac{1+v_{s}}{\mathrm{E}_{\mathrm{s}}}\right) /\left(\frac{1+v_{p}}{\mathrm{E}_{\mathrm{p}}}+\frac{1+v_{s}}{\mathrm{E}_{\mathrm{s}}}\right)\right] .
$$

The physical meaning of the Eq. (5) is simply that the thermal expansion induces not only the stress (strain) along the fiber but also the radial stress, $\mathrm{p}_{\mathrm{r}}$, which arises as the result of the 3-dimensional model. Apparently both are related to the mechanical properties of the two materials and the geometric factor $\gamma$.

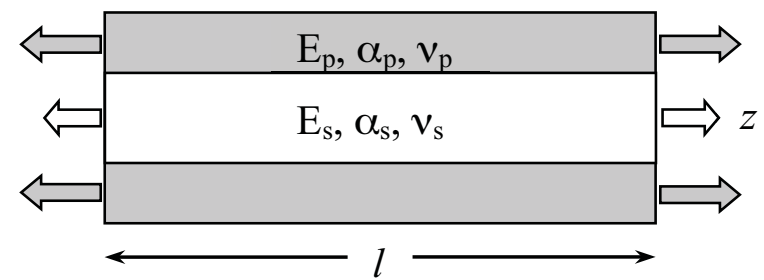

(a)

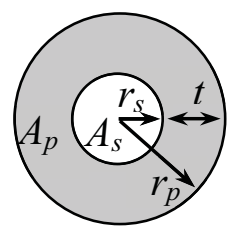

(b)

FIGURE 1. Geometry defining the strain induced by thermal expansion of the fiber and the coating polymer, (a) the side view, and (b) the cross-section view. $\alpha_{p}$ and $\alpha_{s}$ are the thermal expansion coefficients, $E_{p}$ and $E_{s}$ the Young's moduli, $v_{\mathrm{p}}$ and $v_{\mathrm{s}}$ the Poisson's ratios, $r_{p}$ and $r_{s}$ the radii, and $A_{p}$ and $A_{s}$ the cross-sectional areas of the polymer and the silica glass respectively.

It seems the radial stress, $p_{r}$ is missing or at least not explicitly expressed in Eq. (2). To further investigate, one can rewrite Eq. (2) as

$$
\delta \lambda_{\mathrm{B}} / \lambda_{\mathrm{B}}=\left[\alpha_{\mathrm{s}}+\left(1-\mathrm{p}_{\mathrm{e}}\right)\left(\alpha_{\mathrm{p}}-\alpha_{\mathrm{s}}\right) \eta+\xi\right] \Delta T,
$$

where $\xi=\left(1 / \mathrm{n}_{\text {eff }}\right)\left(\partial \mathrm{n}_{\text {eff }} / \partial T\right)$ is the thermo-optic coefficient of the fiber. If there is no coating material, $t=0, \eta=0$, then Eq. (6) becomes the well-know formula for a bare fiber grating sensor,

$$
\delta \lambda_{\mathrm{B}} / \lambda_{\mathrm{B}}=\left(\alpha_{\mathrm{s}}+\xi\right) \Delta T
$$

The difference between Eq. (6) and Eq. (7) is the second term in Eq. (6), the wavelength shift given by the thermally induced strain from the coating. Experimentally one can measure the total Bragg wavelength shifts of FBGs with and without a coating and then compare their difference with the calculated value of the second term in Eq. (6). This can verify whether Eq. (6) represents all of the thermally induced effects or if the induced radial stress, $\mathrm{p}_{\mathrm{r}}$ also contributes to the total wavelength shifts.

\section{EXPERIMENT}

The low (smaller than a few tenths of one percent) reflectivity FBGs used in this research were written in situ, into the drawn optical fiber using the NASA Langley optical fiber draw tower. The drawn fibers with FBGs were coated with polyimide of various thicknesses, and then ink-marked to show grating locations.

These low reflectivity FBGs, written at the same wavelength on a single fiber at different locations, were interrogated using a frequency domain demodulation system [5]. The detected signals from each grating are superimposed and can be fast-Fouriertransformed to obtain the spatial spectrum of all gratings, which displays the physical profiles of the gratings at different locations. The spatial spectrum of a particular grating 
can then be windowed and inverse-fast-Fourier-transformed to get its own wavelength spectrum, as shown in Fig. 2. For the distributed thermal sensing, the center wavelength shifts of each FBG were measured and calculated.

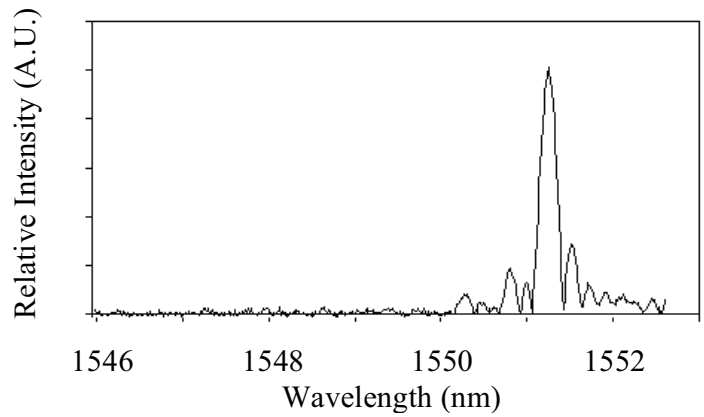

FIGURE 2. The calculated wavelength spectrum of a FBG with a center wavelength of $1551.3 \mathrm{~nm}$.

A cryostat of compressed helium gas was used for temperature control, ranging from $350 \mathrm{~K}$ to about $8 \mathrm{~K}$. The optical fiber with the investigated Bragg grating was held between the cold plate of the cryogenic chamber and another copper plate with a length of about $2 \mathrm{~cm}$ to cover the whole Bragg grating. As shown in Fig. 3, four screws with four identical springs were used to hold the plates and apply a small diametric load to the fiber to form a pressurized Bragg grating (PFBG). A section of support fiber of equal diameter with the test fiber was added to keep the two plates parallel. The total force applied by the springs to the copper plate was approximately $15 \mathrm{~N}$, calculated from the spring constant at room temperature. For the whole range of the temperature change, the total variations of the spring constant and the dimension changes of the copper plates and the steel screws were estimated to be less than a few percent. The applied force was sufficient to reach the threshold stress required for a PFBG but was kept less than $30 \mathrm{~N}$ to prevent splitting of the center peak in the wavelength spectrum [6]. Data were acquired periodically throughout the cooling cycle using the frequency domain demodulation system described previously. The time required to cool from room temperature to the lowest temperature of $8 \mathrm{~K}$ was approximately two hours.

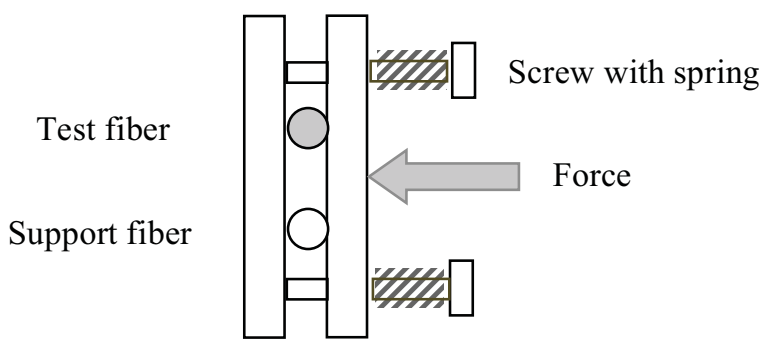

FIGURE 3. Schematic representation of a pressurized fiber Bragg grating (PFBG). A copper plate with four screws holds the test fiber and the support fiber onto the cold plate of the cryogenic chamber. Four springs apply a diametric load to the test fiber to form a PFBG.

For comparison, fiber Bragg gratings with and without coating were also immersed directly in liquid nitrogen to measure their Bragg wavelength shifts.

\section{RESULTS AND DISCUSSION}

In Table 1 , the second column shows the Bragg wavelength shifts, $\Delta \lambda_{B}$, of the FBGs with various coating thicknesses as they were immersed in liquid nitrogen directly 
from room temperature. The third column shows the difference, $\Delta_{C}$, of the wavelength shifts of FBGs with and without the coating. The second term of Eq. (6) were calculated for each FBG of various coating thickness, by using the values from the literature and then subtracted from $\Delta_{C}$. These extra wavelength shifts, $\Delta_{M}$ listed in the fourth column, are attributed to the refractive index change of the optical fiber by the thermally induced radial pressures $p_{r}$ 's as shown in the fifth column.

TABLE 1. The Bragg wavelength shifts and induced radial pressures of FBGs immersed in liquid nitrogen

\begin{tabular}{|l|l|c|c|c|c|}
\hline \multicolumn{2}{|c|}{$\begin{array}{c}\text { FBGs } \\
\text { (with coating thickness, } \mathrm{t})\end{array}$} & $\Delta \lambda_{\mathrm{B}}(\mathrm{nm})$ & $\Delta_{\mathrm{C}}(\mathrm{nm})^{\mathrm{a}}$ & $\Delta_{\mathrm{M}}(\mathrm{nm})$ & $\mathrm{p}_{\mathrm{r}}(\mathrm{MPa})$ \\
\hline 0 & FBG $(\mathrm{t}=0)$ & 1.36 & 0 & 0 & 0 \\
\hline 1 & FBG $(\mathrm{t}=11 \mu \mathrm{m})$ & 1.47 & 0.11 & 0.103 & 5.65 \\
\hline 2 & FBG $(\mathrm{t}=13 \mu \mathrm{m})$ & 1.65 & 0.29 & 0.274 & 6.38 \\
\hline 3 & FBG $(\mathrm{t}=22 \mu \mathrm{m})$ & 1.77 & 0.41 & 0.338 & 8.58 \\
\hline
\end{tabular}

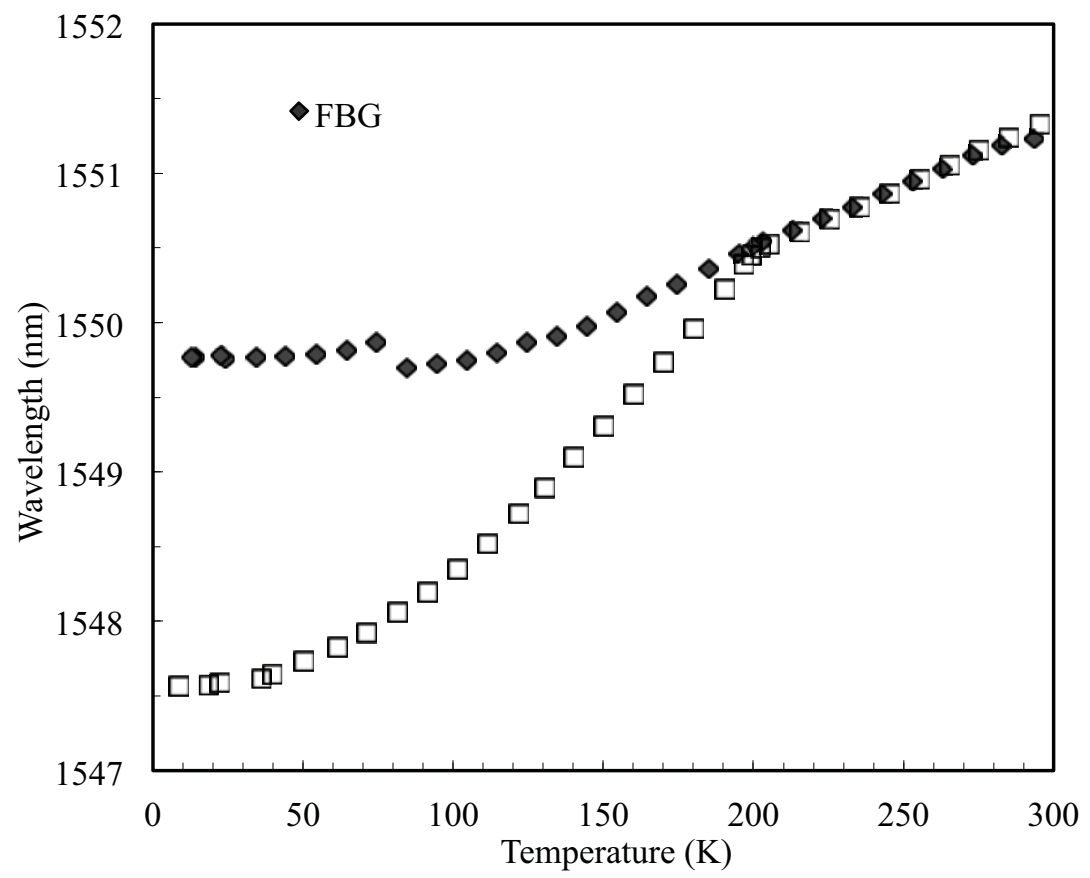

FIGURE. 5. Bragg wavelength shifts of a regular FBG and a PFBG with temperature during a cooling cycle. The coating thickness is $11 \mu \mathrm{m}$ for each.

Figure 5 shows the measured Bragg wavelength versus temperature for a regular FBG with polyimide coating, which has a thickness of about $11 \mu \mathrm{m}$. The FBG was only attached to the cold plate for temperature control without being pressurized. For temperatures from $295 \mathrm{~K}$ to $200 \mathrm{~K}$, the temperature sensitivity of the regular FBG is about $8 \mathrm{pm} / \mathrm{K}$; for temperatures ranging from $200 \mathrm{~K}$ to $80 \mathrm{~K}$ the coefficient is more nonlinear and becomes smaller as the temperature decreases; and for temperatures below $80 \mathrm{~K}$ the curve is almost flat, i.e., showing zero temperature sensitivity. Also shown in Fig. 5 is the measured Bragg wavelength as a function of temperature for the PFBG with the same thickness of polyimide coating. The temperature sensitivity of the PFBG for temperatures 
from $295 \mathrm{~K}$ to $200 \mathrm{~K}$ is about $9 \mathrm{pm} / \mathrm{K}$, not significantly greater than that of the regular FBG. However, at about $200 \mathrm{~K}$, a pressure-induced transition occurs. Below $200 \mathrm{~K}$ the temperature coefficient of the PFBG reaches $24 \mathrm{pm} / \mathrm{K}$, more than three times the regular FBG. Moreover, for the temperature change from $80 \mathrm{~K}$ to $10 \mathrm{~K}$, the PFBG has a total wavelength shift of about $470 \mathrm{pm}, 10$ times more than the regular FBG.

The extra wavelength shift of a PFBG resulting from the pressure-induced transition is apparently attributed to the change of the refractive index, which is related to the impurity (germanium) energy level and the population of the states in the fiber core [7]. These effects are similar to those affecting the energy levels of semiconductors [8], although details of the mechanisms for the transition need further investigation.

\section{CONCLUSION}

We have developed a 3-dimensional physical model to relate the Bragg wavelength shifts of FBGs to the thermal expansion coefficients, Young's moduli, and radial thicknesses of the coating polymers. The experimental results show that temperature sensitivities of FBGs increase with increasing coating thickness. The major part of these extra wavelength shifts is attributed to the refractive index change of the fiber core produced by the thermally induced radial pressure $p_{\mathrm{r}}$. We have also demonstrated the diametric pressure effects on a PFBG. At $200 \mathrm{~K}$, a pressure-induced transition occurs and the temperature sensitivity of the PFBG below the transition temperature reaches three times as large as that obtained with the FBG. For temperatures below $80 \mathrm{~K}$, the PFBG, although showing nonlinearity, has 10 times more wavelength shift than the regular FBG. The increases of the wavelength shifts from FBGs to PFBGs are independent of the coatings thickness and are attributed to the changes of the fiber thermo-optic coefficients.

\section{APPENDIX. Derivation of Eq. (3), the Thermal Strain of A Polymer-Coated Fiber- A 3-Dimensional model}

For a polymer-coated fiber, it is convenient to choose cylindrical coordinates to express strain-stress relations when the temperature changes. In the case of radial symmetry, the total strain along the $z$ direction, $\varepsilon_{z z}$ and the radial direction, $\varepsilon_{r r}$, of the polymer, indexed $p$, and of the silica glass, indexed $s$, can be written respectively as

$$
\begin{gathered}
\varepsilon_{z z}^{p}=\alpha_{\mathrm{p}} \Delta T+\frac{1}{\mathrm{E}_{\mathrm{p}}}\left[\sigma_{z z}^{p}-v_{p}\left(\sigma_{r r}^{p}+\sigma_{\theta \theta}^{p}\right)\right] \\
\varepsilon_{z z}^{s}=\alpha_{\mathrm{s}} \Delta T+\frac{1}{\mathrm{E}_{\mathrm{s}}}\left[\sigma_{z z}^{s}-v_{s}\left(\sigma_{r r}^{s}+\sigma_{\theta \theta}^{s}\right)\right] \\
\varepsilon_{r r}^{p}=\alpha_{\mathrm{p}} \Delta T+\frac{1}{\mathrm{E}_{\mathrm{p}}}\left[-v_{p} \sigma_{z z}^{p}+\left(\sigma_{r r}^{p}-v_{p} \sigma_{\theta \theta}^{p}\right)\right] \\
\varepsilon_{r r}^{s}=\alpha_{\mathrm{s}} \Delta T+\frac{1}{\mathrm{E}_{\mathrm{s}}}\left[-v_{s} \sigma_{z z}^{s}+\left(\sigma_{r r}^{s}-v_{s} \sigma_{\theta \theta}^{s}\right)\right]
\end{gathered}
$$

where $\alpha$ 's are the thermal expansion coefficients, E's the Young's moduli, $v$ 's the Poisson ratios, and $\sigma$ s the induced stresses along each respective direction, of polymer and silica glass respectively. Since there are no external stresses, the first term of each equation is the thermal expansion of the respective material, and the second and the third terms of each are simply the thermal strains induced by the other respective material. Also, since no external forces exist, i.e., the total forces are zero along each direction, one can obtain 


$$
\begin{gathered}
\sigma_{z z}^{s}=-\frac{A_{p}}{A_{s}} \sigma_{z z}^{p} \equiv-\gamma \sigma_{z z}^{p} \\
\sigma_{r r}^{s}\left(r=r_{s}\right)=-\sigma_{r r}^{p}\left(r=r_{s}\right)=\mathrm{p}_{\mathrm{r}},
\end{gathered}
$$

where of $\gamma$ is the ratio of the cross-sectional area, $A_{p}$, of the polymer with a radial thickness of $\left(r_{p}-r_{s}\right)$ to the cross-sectional area, $A_{s}$, of the silica fiber with a radius of $r_{s}$, and $\mathrm{p}_{\mathrm{r}}$ is the stress at the interface.

To reduce the third terms of Eqs. (A.1) - (A.4), i.e., to express $\sigma_{\theta \theta}$ 's in terms of $\sigma_{r r}$ 's (or $\mathrm{p}_{\mathrm{r}}$ ), one can use the equation of equilibrium

$$
\frac{d \sigma_{r r}}{d r}+\frac{\sigma_{r r}-\sigma_{\theta \theta}}{r}=0
$$

and solve the Navier equation

$$
\frac{d^{2} u}{d r^{2}}+\frac{1}{r} \frac{d u}{d r}-\frac{u}{r^{2}}=0
$$

for the radial displacement $u$ by using the boundary conditions of Eq. (A.6). These four terms evaluated at the interface $r=r_{s}$, are obtained as

$$
\begin{gathered}
\sigma_{r r}^{p}+\sigma_{\theta \theta}^{p}=\frac{2 \mathrm{p}_{\mathrm{r}}}{\gamma} \\
\sigma_{r r}^{s}+\sigma_{\theta \theta}^{s}=2 \mathrm{p}_{\mathrm{r}} \\
\sigma_{r r}^{p}-v_{p} \sigma_{\theta \theta}^{p}=\left[\left(1-v_{p}\right)-\left(1+v_{p}\right)(1+\gamma)\right] \frac{\mathrm{p}_{\mathrm{r}}}{\gamma} \\
\sigma_{r r}^{s}-v_{s} \sigma_{\theta \theta}^{s}=\left(1-v_{s}\right) \mathrm{p}_{\mathrm{r}} .
\end{gathered}
$$

If there is no disbonding of the polymer from the silica fiber when the temperature changes, based on the condition of continuity, both strains should be the same at the interface, i.e., Eq. (A.1) equals to Eq. (A.2). It can be expressed as

$$
\left(\alpha_{\mathrm{p}}-\alpha_{\mathrm{s}}\right) \Delta T+\frac{1}{\mathrm{E}_{\mathrm{p}}}\left[\sigma_{z z}^{p}-v_{p}\left(\sigma_{r r}^{p}+\sigma_{\theta \theta}^{p}\right)\right]-\frac{1}{\mathrm{E}_{\mathrm{s}}}\left[\sigma_{z z}^{s}-v_{s}\left(\sigma_{r r}^{s}+\sigma_{\theta \theta}^{s}\right)\right]=0 .
$$

By substituting Eqs. (A.5), (A.9.1) and (A.9.2) into Eq. (A.10) to replace all $\sigma$ 's with $\sigma_{z z}^{s}$ and $\mathrm{p}_{\mathrm{r}}$, one obtains

$$
\left(\alpha_{\mathrm{p}}-\alpha_{\mathrm{s}}\right) \Delta T-\frac{1}{\gamma \mathrm{E}_{\mathrm{p}}}\left[\sigma_{z z}^{s}+2 v_{p} \mathrm{p}_{\mathrm{r}}\right]-\frac{1}{\mathrm{E}_{\mathrm{s}}}\left[\sigma_{z z}^{s}-2 v_{s} \mathrm{p}_{\mathrm{r}}\right]=0 .
$$

For the axial direction, by the same argument, one can combine Eq. (1) and Eq. (2) and substitute Eqs. (A.9.3) and (A.9.4) into it to obtain

$$
\left(\alpha_{\mathrm{p}}-\alpha_{\mathrm{s}}\right) \Delta T+\frac{1}{\gamma \mathrm{E}_{\mathrm{p}}}\left\{v_{p} \sigma_{z z}^{s}+\left[\left(1-v_{p}\right)-\left(1+v_{p}\right)(1+\gamma)\right] \mathrm{p}_{\mathrm{r}}\right\}+\frac{1}{\mathrm{E}_{\mathrm{s}}}\left[v_{s} \sigma_{z z}^{s}-\left(1-v_{s}\right) \mathrm{p}_{\mathrm{r}}\right]=0
$$

The combination of Eq. (A.11) and Eq. (A.12) allows $p_{r}$ to be expressed in terms of the 
axial stress, $\sigma_{z z}^{s}$ i.e.,

$$
\mathrm{p}_{\mathrm{r}}=\left[\left(\frac{1+v_{p}}{\gamma \mathrm{E}_{\mathrm{p}}}+\frac{1+v_{s}}{\mathrm{E}_{\mathrm{s}}}\right) /\left(\frac{1+v_{p}}{\mathrm{E}_{\mathrm{p}}}+\frac{1+v_{s}}{\mathrm{E}_{\mathrm{s}}}\right)\right] \sigma_{z z}^{s} \equiv \zeta \sigma_{z z}^{s},
$$

where $\zeta$ is defined as the ratio of these two stresses. Eq. (A.11) can therefore be reduced as

and then

$$
\left(\alpha_{\mathrm{p}}-\alpha_{\mathrm{s}}\right) \Delta T-\frac{\sigma_{z z}^{s}}{\gamma \mathrm{E}_{\mathrm{p}}}\left[1+2 v_{p} \xi\right]-\frac{\sigma_{z z}^{s}}{\mathrm{E}_{\mathrm{s}}}\left(1-2 v_{s} \zeta\right)=0,
$$

$$
\frac{\sigma_{z z}^{s}}{\mathrm{E}_{\mathrm{s}}}=\left(\alpha_{\mathrm{p}}-\alpha_{\mathrm{s}}\right) \Delta T\left[\frac{\mathrm{E}_{\mathrm{s}}}{\gamma \mathrm{E}_{\mathrm{p}}}\left(1+2 v_{p} \zeta\right)+\left(1-2 v_{s} \zeta\right)\right]^{-1}
$$

Finally, to find the thermal strain on the silica glass induced by the polymer, one can substitute Eq. (A.15) into the third term of Eq. (A.2), i.e., the third term of Eq. (A.14), which is given by

where

$$
\begin{gathered}
\frac{\sigma_{z z}^{s}}{\mathrm{E}_{\mathrm{s}}}\left(1-2 v_{s} \xi\right)=\eta\left(\alpha_{\mathrm{p}}-\alpha_{\mathrm{s}}\right) \Delta T, \\
\eta \equiv\left[1+\frac{\mathrm{E}_{\mathrm{s}}\left(1+2 v_{p} \xi\right)}{\gamma \mathrm{E}_{\mathrm{p}}\left(1-2 v_{s} \xi\right)}\right]^{-1} .
\end{gathered}
$$

\section{REFERENCES}

1. C. R. Giles, "Lightwave applications of fiber Bragg gratings," J. Lightwave Technol. 15, 1391-1404 (1997).

2. A. D. Kersey, M. A Davis, H. J. Patrick, M. LeBlanc, K. P. Koo, C. G. Askins, M.A. Putnam, E. J. Friebele, "Fiber grating sensors," J. Lightwave Technol. 15, 1442-1463 (1997).

3. S. Gupta, T. Mizunami, T. Yamao, and T. Shimomura, "Fiber Bragg grating cryogenic temperature sensors," Appl. Opt. 25, 5202-5205 (1996).

4. T. Mizunami, H. Tatehata, and H. Kawashima, "High-sensitivity cryogenic fiberBragg-grating temperature sensors using Teflon substrates," Meas. Sci. Technol. 12, 914-917 (2001).

5. M. Froggatt and J. Moore, "Distributed measurement of static strain in an optical fiber with multiple Bragg gratings at nominally equal wavelengths," Appl. Opt. 37, 17411746 (1998).

6. R. B. Wagreich, W. A. Atia, H. Singh, and J. S. Sirkis, "Effects of diametric load on fiber Bragg gratings fabricated in low birefringent fiber," Electron. Lett. 32, 1223-1224 (1996).

7. A. Othonos and K. Kalli, Fiber Bragg Gratings: Fundamentals and Applications in Telecommunications and Sensing, Artech House, Boston \& London, 20-43, (1999).

8. J. I. Pankove, Optical Processes in Semiconductors, Dover Publications, New York, 20-28, (1975). 